\title{
Valutazione farmacoeconomica dell'uso di tramadolo vs ketorolac nella gestione del dolore postoperatorio
}

Lorenzo G. Mantovani*

\begin{abstract}
This article represents the first evaluation that compares the economic consequences of managing postsurgical pain with tramadol or ketorolac in the Italian setting.

The economic evaluation was based on 5 clinical trials that directly compared the efficacy and tolerability of tramadol and ketorolac treatments in different settings and that contained drug consumption data.

Since the review of these data confirmed that their efficacy is comparable (tramadol showed a quicker onset of the analgesic action, which was not considered for the purposes of the economic evaluation), it was chosen to conduct a cost minimization analysis. The calculations were made considering two different drug acquisition costs: the hypothetical price for the hospital, computed by discounting $50 \%$ off the retail price, and the mean price really paid by hospitals according to the IHS.

The analyses showed that, when the discounted price is applied, tramadol induces costs that are similar to those induced by ketorolac in $2 / 3$ of the cases and lower in the remaining $1 / 3$. Considering the mean price paid by hospitals, tramadol results economically more advantageous than ketorolac in all considered settings. In conclusion, ketorolac and tramadol are two effective and tolerable drugs in the management of post-surgical pain, although they differ in the adverse event pattern and in the rapidity of their action. In the Italian setting, tramadol appears to be economically more efficient, due to lesser consumption and lower drug acquisition costs.
\end{abstract}

Farmeconomia e percorsi terapeutici 2003; 4 (1): 11-16

\section{INTRODUZIONE}

\section{Farmacoeconomia}

La crescita delle spese sanitarie avvenuta negli ultimi decenni nei paesi a più elevato sviluppo economico è essenzialmente imputabile a tre fattori.

Innanzitutto vi è l'introduzione di nuove tecnologie di diagnosi e cura, generalmente più efficaci ma anche più costose. In secondo luogo si riscontra un progressivo invecchiamento demografico, che provoca un aumento di quella parte della popolazione che maggiormente richiede prestazioni sanitarie; in questo contesto si sono verificate vere e proprie epidemie, particolarmente nell' ambito delle malattie cronico-degenerative. In terzo luogo vi è la crescita delle aspettative della popolazione, con un livellamento verso l'alto dello standard di vita. L'effetto di ciascuno di questi fattori tende a rafforzarsi interagendo con gli altri.

Si può affermare inoltre che uno stimolo fondamentale alla crescita della spesa sia il progresso tecnologico, il quale da un lato fa aumentare la durata della vita della popolazione e dall'altro ne fa crescere le aspettative, in termini di disponibilità, di efficacia e di "comodità" delle tecnologie. Va notato che crescita delle aspettative e invecchiamento della popolazione creano, per parte loro, una spinta verso il progresso tecnologico, attraverso l'allargamento del mercato delle tecnologie mediche.

All'incremento di risorse richieste per la gestione della sanità ha fatto seguito una maggiore attenzione all'uso che di tali risorse viene fatto, e ai relativi costi.

I pazienti, gli operatori sanitari, i cittadini chiedono che le nuove tecnologie siano efficaci nel contrastare le malattie, nel ridurre la disabilità e nel prolungare la vita. Necessariamente però, a
* Centro di

Farmacoeconomia Università degli Studi di Milano 
queste richieste devono corrispondere costi sociali sostenibili e giustificabili.

Compito della farmacoeconomia è quindi la valutazione sotto il profilo economico di più interventi sanitari, di cui almeno uno di carattere farmacologico, per mettere in relazione i costi delle terapie con gli effetti che esse producono.

I decisori pubblici si trovano a dover affrontare scelte riguardanti l' acquisto, l'adozione, la copertura e l'uso delle tecnologie, spesso senza poter disporre degli strumenti adatti a prendere decisioni razionali. Può accadere inoltre che i pazienti facciano pressione sui medici e che questi ultimi si rivolgano agli amministratori pubblici per acquistare mezzi tecnologici sempre più innovativi.

E questo avviene, purtroppo, in una situazione in cui ospedali, aziende sanitarie locali, regioni e $\mathrm{SSN}$ si trovano ad amministrare fondi sempre limitati. C'è il rischio che le decisioni vengano adottate più in modo emotivo che razionale, soprattutto considerando che, di frequente, le informazioni necessarie non sono disponibili. In tale contesto risulta di vitale importanza produrre informazioni solide finalizzate ad assistere i decisori.

\section{Dolore}

Il dolore è un'esperienza comune nei pazienti sottoposti ad intervento chirurgico. Nel $40-60 \%$ dei casi infatti i pazienti definiscono l'intervento come un evento "molto doloroso". Un adeguato trattamento del dolore è necessario per ragioni di carattere non solo umanitario, ma anche medico, al fine di prevenire ed evitare le conseguenze negative sui sistemi endocrino, cardiovascolare e respiratorio, oltre che sul sangue e sullo stato psichico del paziente. L'insorgenza di dolore postoperatorio è ben documentata [1].

\section{Ketorolac e tramadolo}

I farmaci antinfiammatori non steroidei (FANS) occupano una posizione di rilievo tra $i$ farmaci analgesici, inibendo la sintesi delle prostaglandine, responsabili della sensibilizzazione del nocirecettore periferico. Assorbiti rapidamente, sono caratterizzati da ceiling effect e la durata d'azione varia tra le 4 e le 12 ore. Possono provocare trombi e leucocitopenie, oltre alla ben nota gastrolesività. Ketorolac appartiene alla classe dei FANS. La potenza analgesica di $30 \mathrm{mg}$ di ketorolac in somministrazione parenterale è pari a $9 \mathrm{mg}$ di morfina sempre per via parenterale. Ketorolac risulta particolarmente gastrolesivo.

Tramadolo esplica la sua azione analgesica secondo un meccanismo d'azione sinergico: inibisce la trasmissione e la percezione del dolore mediante un'azione agonista sui recettori per gli oppioidi $\mu, \kappa$ e $\delta$ con selettività specifica per i $\mu$ e mediante la modulazione della concentrazione di noradrenalina e serotonina a livello sinaptico centrale. Tramadolo è dotato di un rapido inizio d'azione. Il principale effetto collaterale è costituito dall'insorgenza di nausea e vomito.

\section{MATERIALIEMETODI}

\section{Obiettivo}

L'obiettivo del presente studio è la valutazione farmacoeconomica comparativa di tramadolo $v s$ ketorolac nella gestione del dolore postoperatorio.

\section{Tecnica}

È stata condotta una analisi di minimizzazione dei costi ospedalieri, confrontando i trattamenti alternativi tramadolo e ketorolac.

\section{Costi}

Sono stati considerati essenzialmente i costi dei farmaci. Laddove possibile, è stato inoltre considerato il costo per la gestione degli eventi avversi in termini di consumo di farmaci.

I prezzi dei farmaci sono stati quantificati utilizzando sia lo sconto minimo sul prezzo al pubblico del $50 \%$ da effettuare al momento della cessione dei farmaci ad ospedali (IPOTESIA), sia utilizzando il prezzo medio di vendita ospedaliera di tali farmaci (IPOTESI B), quale risulta dalle delibere di aggiudicazione rilevate e certificate da IHS.

Tutte le analisi si riferiscono a comparazioni di ipotetiche coorti di 100 pazienti per gruppo. I costi sono espressi in euro 2002.

\section{Efficacia}

L'efficacia è stata desunta da 5 studi clinici randomizzati, controllati, comparativi di tramadolo vs ketorolac, individuati e reperiti attraverso una ricerca bibliografica sulle principali banche dati di letteratura medica (Medline ed EMBase).

Si tratta di studi che hanno confrontato ketorolac e tramadolo in differenti tipi di interventi e setting: chirurgia ortopedica, chirurgia maxillo-facciale, chirurgia ginecologica, chirurgia dell'addome superiore.

\section{RISULTATI}

\section{Efficacia}

Sono stati individuati e reperiti in extenso 5 studi clinici randomizzati comparativi di ketorolac $v s$ tramadolo nella gestione del dolore postoperatorio. In tali studi il numero complessivo di soggetti trattati con tramadolo e 
con ketorolac è stato rispettivamente di $162 \mathrm{e}$ di 165 pazienti.

Ketorolac vs tramadolo: studio sull'efficacia analgesica per il dolore postoperatorio di isterectomia addominale [2]

Obiettivo dello studio era confrontare l'efficacia analgesica di tramadolo rispetto a ketorolac somministrato via endovena nelle 24 ore successive ad un intervento di isterectomia addominale.

Questo studio controllato, in doppio-cieco, randomizzato, ha arruolato 76 donne sottoposte ad un intervento di isterectomia addominale, assegnate a due gruppi di trattamento: il gruppo tramadolo, trattato con 100 $\mathrm{mg}$ del farmaco e il gruppo ketorolac, che riceveva per 6 ore $30 \mathrm{mg}$ del farmaco per via endovenosa. Le pazienti, di età compresa tra i 35 e i 65 anni, presentavano un punteggio della scala ASA pari a I-II e venivano escluse dallo studio se l'isterectomia veniva praticata per la presenza di un tumore, se avevano avuto esperienza di discrasie emorragiche, ulcera gastrica o duodenale, se erano allergiche ai farmaci in studio o se nei 15 giorni vicini all'intervento avevano assunto altri farmaci analgesici. L'efficacia dei farmaci in studio è stata valutata adottando una scala analogico visiva (VAS) e una scala di risposta verbale (VRS). Sono state raccolte informazioni anche sull'esigenza di trattamento analgesico, sul numero dei pazienti che interrompevano lo studio prima della fine $\mathrm{e}$ sugli effetti collaterali associati al trattamento.

Il punteggio medio della VAS è risultato di 3.6 per il gruppo tramadolo e di 4.4 per il ketorolac (differenza non significativa, $\mathrm{p}=0,05$ ), punteggio simile a quello rilevato con la scala VRS. Nelle prime 12 ore dopo l'intervento, il punteggio VAS nel gruppo tramadolo era significativamente più basso di quello del gruppo ketorolac $(\mathrm{P}<0.05)$. Nove pazienti hanno abbandonato lo studio, di cui tre nel gruppo tramadolo e uno nel gruppo ketorolac. Tre pazienti del gruppo tramadolo si sono ritirati dallo studio: due per vomito e uno per motivi legati alla somministrazione. Nel gruppo ketorolac sei pazienti hanno abbandonato lo studio: quattro per dolore incontrollabile, uno per impossibilità di intubazione e uno per motivi legati alla somministrazione del farmaco. Non sono state rilevate complicanze postoperatorie importanti.

Durante le prime 12 ore successive all'intervento di isterectomia una dose da $100 \mathrm{mg}$ di tramadolo è risultata più efficace nella riduzione del dolore rispetto a $30 \mathrm{mg}$ di ketorolac somministrato via endovena per 6 ore. L'effetto indesiderato più frequente è stato il vomito postoperatorio, peraltro possibile in chirurgia ginecologica. Nella maggior parte dei casi è stato facilmente controllato con l'utilizzo di metoclopramide $10 \mathrm{mg}$.

Efficacia analgesica del tramadolo

vs ketorolac nella sterilizzazione con

laparoscopia con ricovero di un giorno [3]

È stato condotto uno studio prospettico, randomizzato in doppio cieco per confrontare l'efficacia analgesica del tramadolo somministrato per via endovenosa, $1,5 \mathrm{mg} / \mathrm{Kg}$ e quella del ketorolac $10 \mathrm{mg}$ in 60 pazienti di grado ASA I e II programmati per un ricovero giornaliero per sterilizzazione con laparoscopia. I pazienti sottoposti a trattamento con tramadolo hanno avuto significativamente minor dolore postoperatorio $(\mathrm{p}=0,007)$ e alla dimissione dall' unità operatoria $(\mathrm{p}=0,03)$. Inoltre hanno richiesto aiuto analgesico con morfina meno spesso $(\mathrm{p}=0,02)$ rispetto ai pazienti trattati con ketorolac.

Non è stata rilevata differenza tra i gruppi per quanto riguarda l'incidenza di nausea severa e vomito. Entrambi gli analgesici sono risultati ben tollerati alle dosi somministrate, anche se nei pazienti trattati con tramadolo si è riscontrata più frequentemente secchezza delle fauci ( $\mathrm{p}=0,009)$. Tre pazienti nel gruppo tramadolo e cinque nel gruppo ketorolac hanno richiesto un ulteriore ricovero notturno in seguito al dolore, alla nausea o al vomito.

Trattamento del dolore postoperatorio con PCA nella chirurgia dell'addome superiore: studio comparativo di tramadolo $v s$ metamizolo e ketorolac [4]

L'obiettivo dello studio è stata la valutazione comparativa dell'efficacia analgesica postoperatoria di tramadolo, ketorolac e metamizolo mediante patient contolled analgesia (PCA) più infusione continua nei pazienti sottoposti a chirurgia dell'addome superiore. È stato condotto uno studio clinico randomizzato in doppio cieco che ha incluso complessivamente 100 pazienti, 32 trattati con tramadolo, 37 con ketorolac e 31 con metamizolo. La valutazione dei soggetti è avvenuta in relazione a diversi parametri: il dolore è stato valutato con la Compound Verbal Scale (CVS) e con la Visual Analogue Scale (VAS); sono inoltre stati valutati stato di sedazione, variabili respiratorie, variabili endocrino-metaboliche ed emodinamiche e potenziali effetti collaterali.

Lo studio mostra come tramadolo sia efficace con rapidità maggiore rispetto a ketorolac e metamizolo in termini sia di CVS sia di VAS e abbia un numero inferiore di drop-out attribuibili ad analgesia insufficiente. Né lo stato di sedazione, né le variabili respiratorie, endocrino-metaboliche ed emodinamiche né 
gli effetti collaterali sono invece risultati differenti.

Tramadolo vs ketorolac somministrati intramuscolo in pazienti con dolore ortopedico e traumatologico postoperatorio: uno studio multicentrico comparativo [5]

Uno studio multicentrico, in aperto, randomizzato, della durata di tre giorni, è stato condotto per valutare l'effetto analgesico e la sicurezza terapeutica di tramadolo intramuscolo in confronto a ketorolac intramuscolo in 48 pazienti sottoposti a intervento chirurgico e con dolore postoperatorio valutato con un punteggio di $75 \mathrm{~mm}$ o più su una scala analogico visuale di $100 \mathrm{~mm}$. Tramadolo $(100 \mathrm{mg} / 2 \mathrm{ml}$ fiale) è stato somministrato al bisogno, fino a un massimo di $400 \mathrm{mg} /$ giorno, e ketorolac (30 mg/ $1 \mathrm{ml}$ fiale) fino a $90 \mathrm{mg} /$ giorno. A nove pazienti tra quelli che hanno assunto tramadolo e a sei tra quelli che hanno ricevuto ketorolac è stata

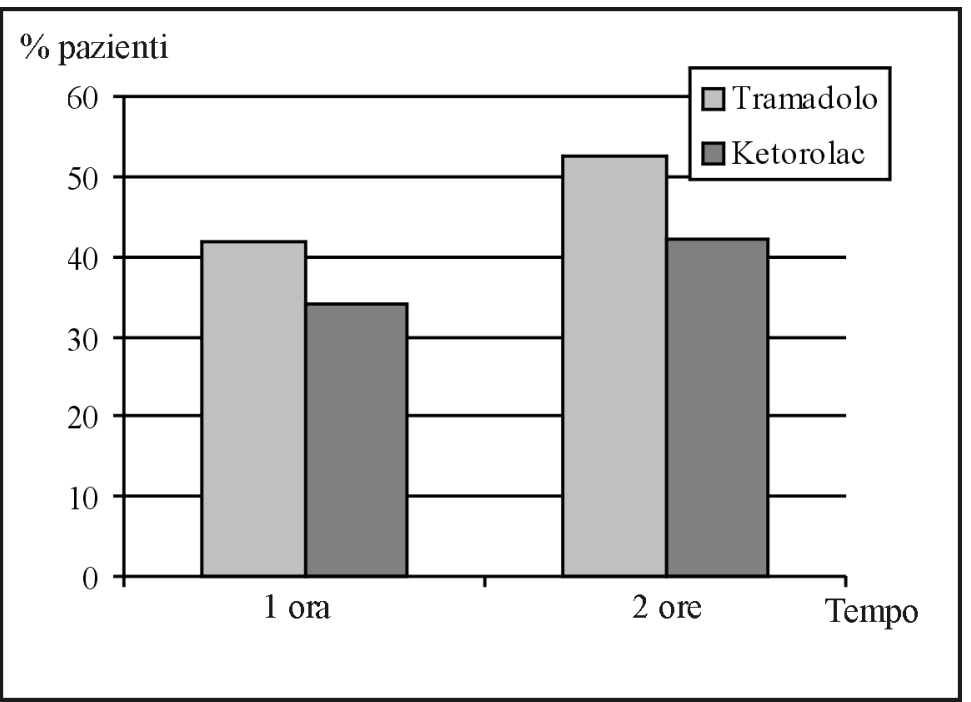

\section{Figura 1}

Effetto analgesico di tramadolo e ketorolac a un'ora e due ore dall'assunzione. (Riferito a dolore ortopedico postoperatorio)

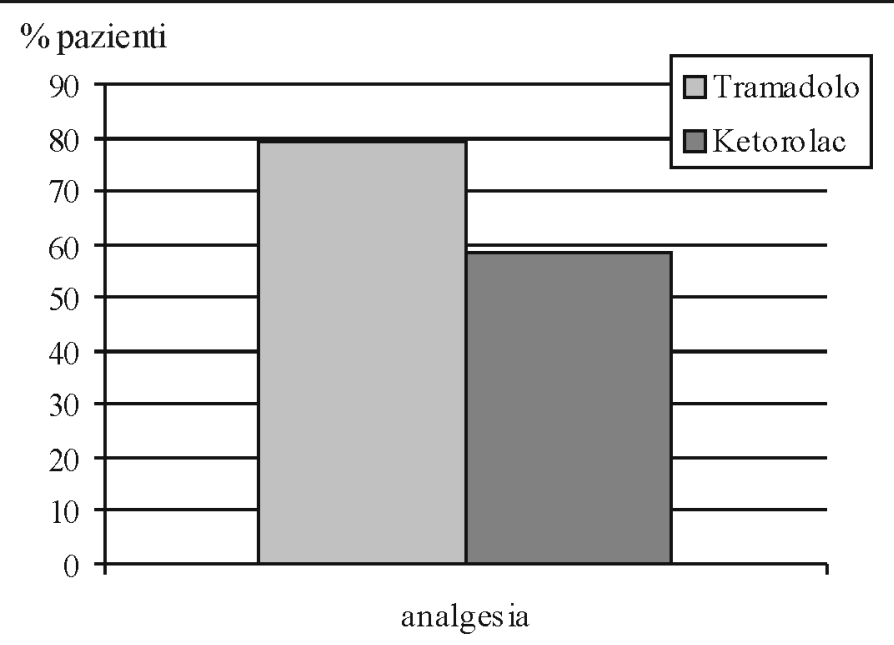

Figura 2

Percentuale di pazienti con analgesia (giudizio finale dei ricercatori) sufficiente una sola dose di farmaco per ottenere un'analgesia soddisfacente.

Tramadolo ha mostrato un effetto analgesico più marcato rispetto a ketorolac. Dopo una e due ore, rispettivamente, il 41,75\% e il $52,5 \%$ dei pazienti che hanno assunto tramadolo hanno trovato sollievo, in confronto al $33,9 \%$ e $42,2 \%$ dei pazienti trattati con ketorolac. Nessuna reazione avversa è stata osservata nei due gruppi e l'opinione finale dei ricercatori è stata positiva: analgesia in 19 pazienti $(79,2 \%)$ sottoposti a trattamento con tramadolo e in 14 pazienti $(58,3 \%)$ trattati con ketorolac. Tramadolo intramuscolo quindi risulta essere efficace almeno quanto ketorolac contro il dolore ortopedico postoperatorio; la tollerabilità è risultata buona.

Tramadolo intramuscolare vs ketorolac nel trattamento del dolore dopo chirurgia nasale: un trial multicentrico controllato [6]

L'effetto analgesico postoperatorio e la tollerabilità terapeutica di tramadolo somministrato per iniezione intramuscolare sono stati confrontati con quelli di ketorolac. Sono stati trattati 77 pazienti sottoposti a chirurgia del naso o dei seni paranasali (39 hanno ricevuto tramadolo, 38 hanno ricevuto ketorolac). L'età media dei pazienti era di $31.0 \pm 10.5$ anni; il peso corporeo era di $69.2 \pm 13.3 \mathrm{Kg}$. Quattro istituti universitari specializzati in otorinolaringoiatria hanno preso parte a questo trial multicentrico, in aperto, randomizzato e controllato. Tramadolo (fiale da $100 \mathrm{mg} / 2 \mathrm{ml}$ ) è stato somministrato al bisogno fino ad un massimo di $400 \mathrm{mg} /$ die, mentre ketorolac (fiale da $30 \mathrm{mg} / \mathrm{ml}$ ) è stato somministrato al bisogno fino a $90 \mathrm{mg} / \mathrm{die}$. La durata del trial è stata di tre giorni.

Entrambi i farmaci hanno dato un controllo rapido e costante del dolore dopo somministrazione della prima fiala e delle successive. La riduzione del dolore e la qualità del sonno (in confronto ai giorni precedenti) hanno mostrato miglioramenti simili; il profilo di sicurezza è stato rassicurante, con solo due eventi avversi tra i pazienti trattati con tramadolo (sudorazione moderata, uno; leggera sudorazione e nausea, uno) e un evento avverso tra quelli trattati con ketorolac (dolore e reazione cutanea nel sito d'inoculazione). Tramadolo IM è risultato efficace nel trattamento del dolore conseguente a chirurgia nasale, tollerabile e maneggevole.

\section{Effetti collaterali}

Negli studi presi in considerazione, tramadolo e ketorolac hanno dimostrato un profilo di tollerabilità sostanzialmente simile.

La terapia con metoclopramide $10 \mathrm{mg}$ è stata in grado di controllare nausea e vomito nella 


\begin{tabular}{ccc}
\hline \multicolumn{1}{c}{ Farmaco } & Prezzo per fiala & Fonte \\
\hline Metoclopramide $10 \mathrm{mg}$ & 0.11 & Informatore Farmaceutico '02 \\
Ipotesi A (50\% sconto obbligatorio) & & \\
Tramadolo $100 \mathrm{mg}$ & 0.88 & Informatore Farmaceutico '02 \\
Ketorolac $30 \mathrm{mg}$ & 0.85 & Informatore Farmaceutico '02 \\
Ipotesi $B$ & & \\
Tramadolo $100 \mathrm{mg}$ & & \\
Ketorolac $30 \mathrm{mg}$ & 0.354 & IHS (rilevazione giugno '02) \\
\hline
\end{tabular}

\begin{tabular}{lccc}
\hline \multicolumn{1}{c}{ Studio } & $\begin{array}{c}\text { Costo gruppo } \\
\text { tramadolo (euro) }\end{array}$ & $\begin{array}{c}\text { Costo gruppo } \\
\text { ketorolac (e uro) }\end{array}$ & $\begin{array}{c}\text { Diffe renza } \\
\text { (e uro) }\end{array}$ \\
\hline Ollè Furtuny et al [2] & 444 & 426 & 18 \\
Colletti et al [6] & 336 & 328 & 8 \\
Lanzetta et al [5] & 639 & 765 & -126 \\
\hline
\end{tabular}

totalità di coloro che hanno terminato lo studio di Ollè Fortuny et al [2], quello in cui è stata evidenziata la maggior insorgenza di questo effetto collaterale nei soggetti randomizzati a ricevere tramadolo. In tal caso, sono stati considerati anche i costi della terapia antiemetica con metoclopramide.

\section{Costi} del prezzo di tramadolo e di ketorolac. Nella prima analisi è stato considerato il costo attuale dei farmaci al pubblico, diminuito dello sconto del $50 \%$ obbligatorio per le forniture ospedaliere. In tal caso i prezzi sono risultati pari a euro 0,88 per fiala di tramadolo $100 \mathrm{mg}$ e euro 0,85 per fiala di ketorolac $30 \mathrm{mg}$. medi praticati in ospedale, pari a euro 0,354 per fiala di tramadolo $100 \mathrm{mg}$ e euro 0,785 per fiala di ketorolac $30 \mathrm{mg}$. $10 \mathrm{mg}$ è stata quantificata al prezzo di euro 0,11 per fiala.
Sono state effettuate due differenti analisi

Nel secondo caso sono stati usati i prezzi

La terapia antiemetica con metoclopramide

\section{Analisi di minimizzazione dei costi}

Ai fini della quantificazione economica sono stati utilizzati i dosaggi effettivamente adottati nei diversi trial descritti sopra e sono stati espressi come costo per 100 pazienti trattati.

Nello specifico, lo studio di Colletti et al [6] ha evidenziato un utilizzo di 386 fiale nel gruppo trattato con ketorolac e di 382 nel gruppo trattato con tramadolo. A tali consumi corrispondono costi totali pari a euro 328 per ketorolac ed euro 336 per tramadolo nell' ipotesi A (sconto 50\%); euro 303 per ketorolac ed euro 152 per tramadolo nell'ipotesi B (prezzo medio effettivo). La terapia con tramadolo è minimamente più costosa nell'ipotesi $\mathrm{A}(+8$ euro), mentre conduce a notevoli risparmi nell'ipotesi B (-151 euro) rispetto a ketorolac.

Sulla base delle informazioni riportate da Lanzetta et al [5], è stato possibile stimare un utilizzo di 900 fiale nel gruppo trattato con ketorolac e di 726 nel gruppo trattato con tramadolo. A tali consumi corrispondono costi totali pari a euro 765 per ketorolac ed euro 639 per tramadolo nell'ipotesi A (sconto 50\%); euro

\begin{tabular}{lccc}
\hline \multicolumn{1}{c}{ Studio } & $\begin{array}{c}\text { Costo gruppo } \\
\text { tramadolo (euro) }\end{array}$ & $\begin{array}{c}\text { Costo gruppo } \\
\text { ketorolac (euro) }\end{array}$ & $\begin{array}{c}\text { Differenza } \\
\text { (euro) }\end{array}$ \\
\hline Ollè Furtuny et al [2] & 181 & 392 & -211 \\
Colletti et al [6] & 152 & 303 & -151 \\
Lanzetta et al [5] & 257 & 706 & -449 \\
\hline
\end{tabular}

\section{Tabella 1}

Costi Unitari (Euro)

Tabella 2.

Analisi di minimizzazione dei costi per 100 pazienti trattati sotto ipotesi A: condotta utilizzando $i$ prezzi di tramadolo $100 \mathrm{mg}$ (euro $0.88 \mathrm{per}$ fiala) e di ketorolac 30 mg (euro 0.85 per fiala), calcolati sulla base dello sconto obbligatorio del $50 \%$.

\section{Tabella 3}

Analisi di minimizzazione dei costi per 100 pazienti trattati sotto ipotesi B:

condotta utilizzando $i$ prezzi medi di vendita in ambito ospedaliero di tramadolo $100 \mathrm{mg}$ (euro 0.354 per fiala) $e$ di ketorolac $30 \mathrm{mg}$ (euro 0.785 per fiala), calcolati sulla base dei prezzi di aggiudicazione rilevati e certificati da IHS. 


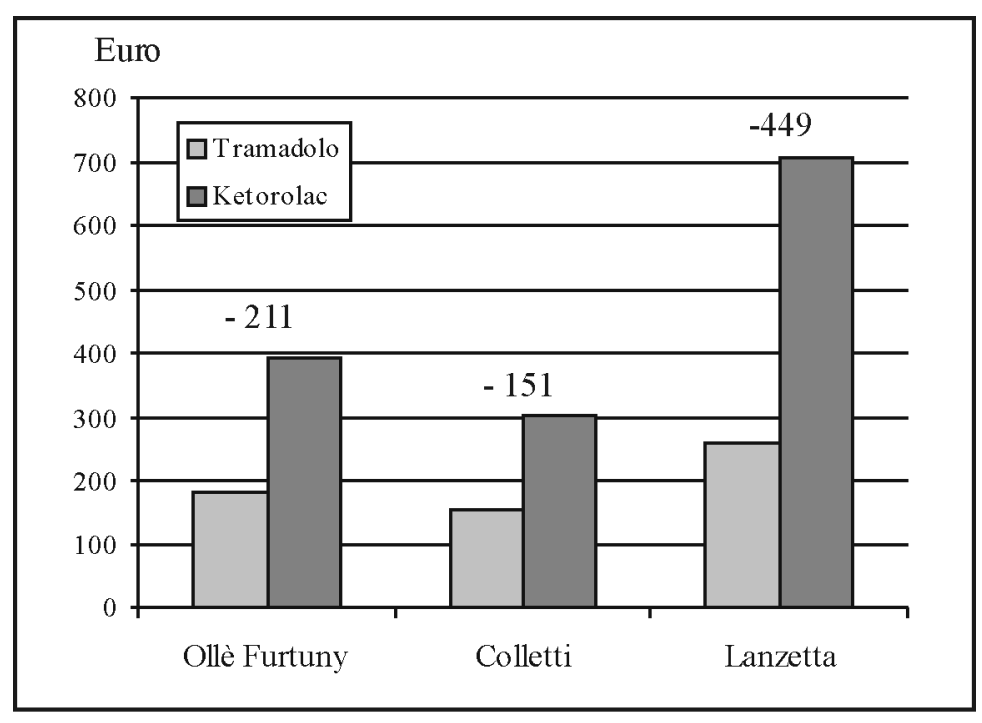

Tabella 3

Analisi di minimizzazione dei costi per 100 pazienti trattati sotto ipotesi B

\section{DISCUSSIONE}

Il presente studio rappresenta la prima valutazione farmacoeconomica comparativa di ketorolac e tramadolo nella gestione del dolore postoperatorio condotta in Italia. Data la dimostrata sovrapponibilità dell'efficacia di tramadolo e ketorolac, impiegati agli opportuni dosaggi (in generale $100 \mathrm{mg}$ di tramadolo e $30 \mathrm{mg}$ di ketorolac per somministrazione), è stata utilizzata l'analisi di minimizzazione dei costi. Nella presente valutazione non è stata invece presa in considerazione la maggior rapidità di azione di tramadolo rispetto a ketorolac.

Dalle analisi condotte è risultato che, nell'ipotesi A (prezzo al pubblico decurtato dello sconto obbligatorio del 50\%), la terapia con tramadolo presenta costi simili al trattamento con ketorolac in due casi su tre. In quest'ultimo caso, che corrisponde allo studio di Lanzetta et al [5], risulta invece decisamente meno costosa, grazie al minor consumo complessivo di tramadolo. Va peraltro notato come l'ipotesi A rappresenti, in questo settore, un caso meramente teorico, poiché vi sono prove che nella realtà gli sconti praticati sono spesso ben maggiori, soprattutto per tramadolo.

Considerando invece l'ipotesi B (prezzo medio realmente praticato in ambito ospedaliero, come rilevato e certificato da IHS), le analisi condotte mostrano come la terapia con tramadolo sia decisamente meno costosa rispetto a quella con ketorolac, in tutti i setting considerati. maggiore nel gruppo tramadolo rispetto a quello ketorolac, ha generato costi di trattamento con metoclopramide, pari a circa euro 5 ed euro 1 rispettivamente. I costi totali sono così risultati di euro 426 per ketorolac ed euro 444 per tramadolo nell'ipotesi A (sconto 50\%); euro 392 per ketorolac ed euro 181 per tramadolo nell'ipotesi B (prezzo medio effettivo). Rispetto a ketorolac, la terapia con tramadolo è minimamente più costosa nell'ipotesi $\mathrm{A}$ (+18 euro), mentre conduce a notevoli risparmi nell'ipotesi B (-211 euro).

\section{CONCLUSIONI}

Tramadolo e ketorolac si dimostrano farmaci efficaci e tollerabili nella gestione del dolore postoperatorio. Tramadolo mostra un'azione analgesica più rapida rispetto a ketorolac $\mathrm{e}$, in virtù nel minor costo di acquisizione e del minor consumo, appare economicamente più efficiente.

\section{BIBLIOGRAFIA}

1. Berti M. Dolore Post-operatorio: la teoria e la pratica. CEDIS, Roma, 1999

2. Ollè Fortuny G, Opisso Julia L, Oferil Riera F et al. Ketorolaco frenete a tarmadol: estudio comparativo de la eficacia analgesica en el dolor postoperatorio de hysterectomias abdominales. Rev Esp Anestasiol Rianim 2000; 47:162-67.

3. Putland AJ and Mc Cluskey A. The analgesic efficacy of tramadol versus Ketorolac in day case laparoscopic sterilisation. Anaesthesia 1999; 54: 372-385.

4. Castro F, Pardo D, Mosquera R, et al. Tratamiento del dolor postoperatorio con PCA ec cirurgia del abdomen superior: estudio comparativo, tramadol versus metamizol y Ketorolaco. Rev Soc Esp Dolor 2000; 7: 12-16.20

5. Lanzetta A, Vizzardi M, Letizia G, et al. Intramuscular tramadol versus Ketorolac in patients with orthopedic and traumatologic postoperative pain: a comparative multicenter trial. Curr Ther Res 1998; 59: 39-47.

6. Colletti V, Corner M, Vincenzi A, et al. Intramuscular tramadol versus Ketorolac in the treatment of pain following nasal surgery: a controlled multicenter trial. Curr Ther Res 1998; 59: 608-618. 